\title{
Healthcare providers' perceptions on screening for Intimate Partner Violence in healthcare: A qualitative study of four health centres in Uganda
}

\author{
Stephen Lawoko ${ }^{1,2^{*}}$, Gloria K. Seruwagi ${ }^{3}$, Iryne Marunga ${ }^{4}$, Milton Mutto ${ }^{4}$, Emmanuel Ochola ${ }^{5}$, \\ Geoffrey Oloya ${ }^{6}$, Joyce Piloya ${ }^{7}$, Muhamadi Lubega ${ }^{8}$ \\ ${ }^{1}$ Department of Public Health Sciences, Karolinska Institutet, Stockholm, Sweden; \\ *Corresponding Author: Stephen.lawoko@ki.se \\ ${ }^{2}$ Makerere University, Kampala, Uganda \\ ${ }^{3}$ Victoria University, Kampala, Uganda \\ ${ }^{4}$ Pincer Group International, Kampala, Uganda \\ ${ }^{5}$ Lacor Hospital, Gulu, Uganda \\ ${ }^{6}$ Anaka Hospital, Gulu, Uganda \\ ${ }^{7}$ Gulu Referral Hospital, Gulu, Uganda \\ ${ }^{8}$ Iganga Hospital, Iganga, Uganda
}

Received 20 September 2012; revised 25 October 2012; accepted 5 November 2012

\section{ABSTRACT}

The current qualitative study explored the perceptions of healthcare providers on screening for Intimate Partner Violence (IPV) in healthcare in Uganda, to develop a conceptual framework for factors likely to hinder/promote IPV screening in the country. Using purposive sampling, the study enlisted 54 healthcare workers (doctors and nurses) from four hospitals (i.e. Gulu referral hospital, Iganga referral hospital, Lacor hospital, Anaka hospital) to participate in eight focus group discussions. Data was thematically analysed using Template Analysis. The study found support for an ecological framework suggesting a complex interaction of factors at the individual (e.g. poor skills in detection of IPV by health workers and unwillingness to disclose abuse by patients), organisational (e.g. understaffing and lack of protocols for IPV screening) and societal (e.g. societal acceptance of abuse of women and poor policy on IPV management) levels as potential barriers to the practice of IPV screening in healthcare Uganda. These findings have important implications on further training of healthcare workers to adequately screen for IPV, re-organisation of the healthcare system so that it is fully-fledged to accommodate IPV screening and improved collaboration between the health sector and other community advocates in IPV management. These initiatives should run concurrently with a concerted community sensitization effort aimed at modifying attitudes towards IPV among care providers and recipients a like, as well as preparing the general population to will-fully disclose IPV to healthworkers. Study limitations and implications for further research are discussed.

Keywords: Intimate Partner Violence; Screening; Healthcare; Perceptions; Uganda

\section{INTRODUCTION}

Intimate Partner Violence (IPV), defined as behaviors within an intimate relationship that cause physical, sexual or psychological harm, including acts of physical aggression, sexual coercion, psychological abuse and controlling behaviors [1], remains a global health problem among women. Despite the inaction of laws and policies to manage Intimate Partner Violence in several countries, IPV prevalence remains alarmingly high globally, with devastating short and long term consequences on women's health. With small cross-country variations, prevalence of IPV ranging between 3\% - 38\% have been reported [1-3], and many of the victims exhibit health and behavioural complications manifest in severe physical injuries, depression, anxiety, post-traumatic stress disorder, suicidal ideation, unhealthy feeding habits, substance abuse and alcoholism [1-8]. Furthermore, victims of IPV in their reproductive age evidence terminated pregnancies, undesired pregnancies and child loss during infancy to a higher degree than peers in non-violent intimate relationships $[1,8,9]$. Thus, IPV does not only impact on abused women's health per se, but is also associated with a poor foetus prognosis and infant 
health.

Despite the poorer health outcomes, IPV victims under-utilize healthcare services and exhibit poorer bonding with healthcare providers $[9,10]$, when contrasted with other healthcare users in general. These findings have important implications for further adaptation of the healthcare system to a more "user-friendly" system for abused women. Screening for IPV is seen as a step in that direction.

Screening for IPV in healthcare requires the systematic involvement of healthcare workers in the detection of IPV among clients who may or may not present with direct signs of victimization/abuse [11]. The discussion on whether or not the practice of inquiry about the possibility of IPV among all women visiting healthcare regardless of the reason for their visit (i.e. universal screening) is on-going, despite reports underscoring the significance of such practice. First, anecdotal evidence suggests that merely recognising and validating a battered woman's situation may have far reaching effects on her response to treatment and ultimately her health [11]. Second, healthcare professionals acknowledge that routine screening is likely to improve identification of IPV [12]. Reciprocating this view, female patients visiting healthcare report increased satisfaction with care, when the issue of IPV is incorporated in health inquiries [13]. A consensus between the stakeholders notwithstanding, only between $8 \%-10 \%$ of healthcare personnel routinely screen for IPV $[14,15]$, suggesting the presence of barriers. The reasons for this discrepancy have over the past decade received increased attention and can be characterised under factors incumbent in care consumption, care provision, care system and society at large. Among female care consumers, the lack of willingness to disclose abuse poses significant threat to effective screening for IPV. Despite the fact that IPV accounts for significant portion of the total care burden [9,16-20], data from low, middle and high income societal contexts suggest that between $20 \%-70 \%$ of women are hesitant to disclose abuse to formal institutions including healthcare [1,21-24]. Pertinent reasons why women may not disclose IPV include the perceived lack of confidentiality and inappropriate methods of inquiry from care providers [25]. Moreover, women in some societies (e.g. low income countries) appear to endorse IPV in certain circumstances, raising important questions as to whether such women would see the significance of IPV screening in healthcare. Studies have for instance found women in Sub-Saharan African countries to endorse wife abuse for reason including failures in her normative domestic roles such as cooking and childcare [26,27]. Among care providers, insufficient knowledge and training in screening could explain the poor frequency in IPV inquiries [28-30]. In addition, professional roles governing the provider-client relations (e.g. mutual respect, fear of offending clients etc.), healthcare provider's individual attitudes towards IPV and cultural values could conflict with IPV inquiry in healthcare [31,32]. At the organisational level, lack of clear policy surrounding IPV management, environmental factors (e.g. privacy in the waiting room, length of wait for the service and lack of continuity of service), and poor availability of protocols for the purpose render the practice of screening difficult [33,34].

In summary therefore, a number of factors at the individual, organisational (care system) and community level appear to affect IPV screening in healthcare. While there is a myriad of data to support these conclusions, researchers acknowledge that some of these factors may be context specific and may not be exhaustive of barriers to screening for IPV in all contexts [32,35], drumming for an in-depth analysis of each unique context.

Qualitative methods have long been proposed as ideal for an in-depth understanding of health attitudes, knowledge and behaviours. Using broad open-ended and interconnected questions, the researcher aims at attaining in-depth insight into an area of interest [36,37]. Though much is now known about the factors that may foster or hinder IPV screening in healthcare in the high income countries, analysis of such factors in new contexts (e.g. low and middle income countries) may exhibit a set of new factors, while not refuting those already known and could be universal. Given such circumstances, template analysis, a qualitative approach, is the preferred tool. This analysis takes into account already existing knowledge in the field, while leaving room for new knowledge to emerge. Further, the method is flexible to refute the application of already existing knowledge to the new context studied (see methodology section for more details).

In this paper, we sought an in-depth understanding of the views of health care providers in Uganda about screening for IPV, to develop a conceptual framework for factors likely to hinder/promote IPV screening in healthcare in Uganda. Such data is deemed important for incorporation in any training program to improve care providers' readiness to screen for IPV. More precisely, the study attempts to answer the following broad questions:

1) What are the views of healthcare providers in Uganda regarding IPV in general in Uganda.

2) What are the views of healthcare providers in Uganda regarding screening for IPV in healthcare?

3) What do healthcare providers in Uganda identify as factors that may hinder/promote IPV screening in healthcare Uganda?

The care-providers views/perceptions will be sought in regard to the following actors 1 ) health consumer; 2) 
health provider; 3) health system; 4) any other party that may emerge during the discussion.

\section{METHODOLOGY}

\subsection{Sampling, Procedures and Participants}

This study uses qualitative methods to understand care providers views and perceptions on screening for IPV in healthcare Uganda. It is part of a larger study comprising both quantitative and qualitative methods to explore possible hinders and enablers of screening for IPV in healthcare Uganda.

The primary method of data collection used in this qualitative arm of the larger study was focus group discussions (FGDs) with frontline health workers. Purposive sampling was undertaken to enlist 54 participants from four (4) hospitals (i.e. Gulu referral hospital, Iganga referral hospital, Lacor hospital, Anaka hospital) in eight (8) focus group discussions. The inclusion criteria were being an enrolled or registered nurse and doctor. These staff cadres where of interest mainly because of their daily interaction with potential IPV victims and their potential integral role in addressing the IPV problem through a screening intervention, being at the forefront of care provision. Exclusion criteria were being other health worker with little or no interaction with potential victims of violence. Semi structured focus group discussion guides were used to elicit information that would answer the underlying research question on health worker perceptions and attitudes towards IPV screening. Separate FGDs were held for each staff cadre and hospital respectively. The FGDs were led by trained moderators and in English, the official language in the country. Discussions were audio-recorded following consent of the participants.

The data collection process, including the sampling procedure, was based on the need to meet the study's primary objective which was to "assess healthcare professionals' attitude towards and perceptions about IPV screening".

Results were discussed on a regular basis by all authors as they emerged. Thus all authors provided vital input for the discussion of this work.

\subsection{Data Analysis}

Data were thematically analyzed using the template analysis (TA) approach $[38,39]$. Template analysis is an adaptation of thematic analysis in qualitative research and has been successfully applied in the field of qualitative research with human subjects in several studies [40-43]. The continued use of Template Analysis to interpret textual data demonstrates its applicability, efficacy and ease of usage in a variety of fields. In addition, Template Analysis has been commended for its flexibil- ity as shown below:

...the template approach allows codes and categories to be presented hierarchically to aid the analytical process in categorising and unitizing data ...while bearing some resemblance to grounded theory, template analysis is less prescriptive and more flexible in its approach to analysis, allowing the researcher to amend its use to the needs of the research project...the template analysis introduces structure and consistency into the categorising and unitizing of qualitative in-depth interview transcripts [41, cited in 40].

Other studies [43] that have used template analysis have concurred with this view that in comparison with grounded theory, template analysis is more flexible and easy to adapt "without necessarily applying puristic inductive procedures and it also offers the prospect of developing broad conceptual themes which can be clustered into broader categories” [40].

The method is particularly useful to study phenomena for which broad themes have already been determined. For instance, with regard to IPV screening, it is known that factors likely to hinder/promote IPV screening in healthcare are likely to be traced to individual, system and societal factors (see introduction for a literature review). This ecological structure thus formed the main themes, from which subsequent smaller levels can be derived.

The main strategy in TA thus is to determine the main themes and derive subsequent smaller level themes in a nested but orderly hierarchical way [38]. The coding template therefore incorporated a number of broader themes a priori which were strongly expected to emerge from the focus group discussions (i.e. in accordance with the ecological model). However, these themes and related codes were subject to modification.

Following identification of a priori themes and their codes, all transcribed focus group discussions were read through and matched to the research questions and existing thematic codes. After reading through and marking all the transcripts then this template was applied to the whole dataset and transcripts which were then coded to it. This modified and all-inclusive template was then used as the basis for interpretation, analysis and writing up of the research findings. The initial coding template was developed using information from the research questions and FGD guide which were developed prior to the commencement of fieldwork. In its development, we were cognizant of new emerging themes that were not originally anticipated and these were incorporated in the coding template as the FGD transcripts were read through and assigned to (either a priori or new) codes.

Whilst Template Analysis was used to guide analysis this study was aware that it is only a helpful tool for organising data. Actual interpretation of the data was 
guided by the aims and objectives of this study, the guiding research questions, as well as the researchers' epistemological position and assumptions.

\subsection{Ethical Considerations and Approval}

For all participants informed Consent was given. Participants were informed of the nature of the research (goals and objectives, etc.), of the research methodology to be used, of any risks or benefits, of their right not to participate and /or to terminate participation at any time. In addition, anonymity and confidentiality were emphasized. The study was approved by the Makerere University Research Ethics Committee and the Uganda National Council for Science and Technology, the two bodies responsible for ethics in scientific research in Uganda.

\section{RESULTS}

\subsection{Views on IPV in General}

Health workers generally associated IPV with domestic violence and observable physical harm in particular. The most common forms of IPV they confronted in healthcare were physical violence and sexual abuse, including marital rape. In some cases it involved extreme cases of murder as shown in the excerpt below:

The most common forms of IPV we come across are domestic violence mainly between husbands and wives, and forced sex between lovers when one party is not in agreement (Doctor, Anaka).

It is physical fighting between lovers, sometimes... killing of the whole family (Nurse, Anaka).

Men are being beaten these days but still the most common one is the women being beaten (Nurse, Gulu).

We usually know at the time of treating injuries...especially when people have police forms or severe bruises, or they seem emotionally unstable (Nurse, Anaka).

As shown in the excerpts above, health service providers used a clinical lens to conceptualize IPV. Their understanding was directly related to their clinical duties at the frontline and was closely linked to the responses that victims required at health facilities in the aftermath of IPV such as treatment for injuries incurred.

IPV was found to be largely engendered with men being the main perpetrators against women although women were also noted to inflict violence on their partners. Evolving trends in IPV were also noted by health workers particularly abdication of family roles by men and women inflicting violence on men.

Adultery for women is punishable but not for men (Doctor, Iganga hospital).

Husbands ignore mothers and their children and even use guns which was not the case back then (Nurse, Iganga hospital).
Things are changing...there are also women who batter their husbands and send them away from their own homes (Doctor, Lacor hospital).

It comes in form of misunderstanding of women by the men most especially the nurses as their husbands think they don't want to give birth (Nurse, Iganga hospital).

The excerpts above show that IPV is disproportionately experienced by women compared to men and this is largely due to perceptions that are rooted in sociocultural values in patriarchal societies that tend to favour men. Whereas there was some awareness around linking IPV to other subtle forms such as men abandoning their families, this was blurred by more explicit forms of violence such as wife beating and using guns.

Health workers attributed some of the IPV evolving trends (e.g. women as perpetrators) to recent movements such as women "emancipation" and the rights movement which strains relationships and appears to challenged long standing and deeply cherished cultural values of female compliance to males as shown below:

Women emancipation has made women more confident and not respectful of their husbands (Doctor, Iganga hospital).

IPV occurred as a result of cultural tensions where deeply valued norms and traditions were being challenged for example imbalance in the dynamics of power at familial level as well as childbirth which was being overlooked by women in pursuit, or as a result, of their careers. It also shows the widespread nature of IPV as health service providers are themselves victims. Health care professionals are not immune to IPV and in addition to having a responsibility to address IPV issue with their clients, they are victims themselves. Some of the health care professionals were upfront about their victim status as seen from the excerpts below:

Even health workers are burdened. They are also victims of IPV (Doctor, Iganga).

In the case of marital rape we are all being raped as women especially when a man comes home drunk then starts forcing himself on you when you already resting. In the end we may fight or I will get beaten because I will not allow you to enter me. so in this case one is not allowed to report (Nurse, Gulu Hospital).

This study found societal acceptance towards some forms of IPV and it was noted that the lived experience of some IPV victim is one of acceptance and positive perceptions. The most commonly "acceptable" form of IPV was found to be wife beating whose function was delineated to be two-fold: In the first instance, wife beating was interpreted as a sign of love and was well received by some women:

Wife beating is culturally accepted. It is a form of discipline. Some women complain when their husbands don't beat them because it is a sign of love (Nurse, 
Anaka hospital).

Secondly, wife beating was also reported to be a disciplinary measure used by the head of the household within a home setting:

It is acceptable for example a man beating a wife for the wrong she has done. It is a way of "correcting" or disciplining her (Doctor, Anaka hospital).

Some men use wife beating as a form of discipline to the wife. This is acceptable if there is no injury or not life threatening...a man is entitled to discipline his wife (Nurse, Gulu hospital).

Cultural beliefs were highlighted as a major reason for IPV and it accounted for unabated levels of some IPV forms such as the one mentioned above.

Fighting in some cultures is tolerated as it's a sign of love and some women are taken as property in the home (Nurse, Iganga hospital).

Rape in marriage is so much tolerated in this community because culturally when a woman is married, she is expected to satisfy the man with sex at any time he wants cause it's the main reason for her getting married (Doctor, Iganga hospital).

Beating a wife is tolerated in society, this is because women have been made to be inferior, especially in Acholi culture, they say, "a mere woman", you don't have any say, so women have taken that saying to be right. So for any slight thing you are beaten even in front of your children. So it is accepted. (Nurse, Gulu hospital).

As shown in the excerpts some forms of IPV such as physical violence and marital rape were found to be not only accepted but also encouraged. Other key triggers of IPV reported by discussants included poverty, high sexual drive among men, ignorance, illiteracy, infidelity and high risk behaviour such as alcoholism and substance abuse.

In Northern Uganda, IPV was also found to be as a result of challenges of resettling former Internally Displaced People (IDP) into their communities. It was clear that scramble for land and meagre resources in the aftermath of war had led to a number of conflict. Study participants also reported that it is mainly women who bore the brunt of the violence and injustice both from their partners as well as their partners' families as shown below:

After the war most cases nowadays are related to land disputes, you find that a widow has come back to the village and now wants to settle, but cannot get back their land and the man's family tends to chase away the woman especially when they were not married they think she has been away with other me (Doctor, Lacor).

Societal response to IPV cases were found to be weak and not protective of the victims which partly accounts for the lack of willingness on the part of the victims to disclose or report:

I will give you an example about my real sister. He [husband] had a misunderstanding with the wife. The woman had just delivered and the man wanted to have sex. The woman refused and said "I have just given birth". The man sent away the woman with all the six children. The woman's brother called him to settle the matter but he refused. When they reported him to police the family and protection unit, the man was summoned, and asked to take back his wife and children and take care of them (Nurse, Gulu Hospital).

Police is not helping because they ask for money from victims even when they can't afford. So they end up not reporting because they don't have confidence in police (Doctor, Iganga Hospital).

These days people rarely report so they die quietly (Doctor, Lacor Hospital).

As shown above system inefficiencies were found to have resulted into frustration and resignation on the part of IPV victims and rendered them voiceless, further perpetuating the cycle of IPV.

\subsection{Views on Healthcare Workers Capacity to Screen/Involvement in IPV Screening}

Health workers assessed themselves as being capable of providing IPV screening and their perception towards the intervention was positive. Most discussants looked at IPV as a legitimate issue that should be addressed. The FGD excerpts from below shows this:

Health workers should screen for IPV and it should be part of our main duties as there are many cases of IPV (Nurse, Iganga hospital).

Screening IPV is necessary and it should be mainstreamed in the daily duties (Doctor, Iganga hospital).

Screening IPV is necessary and it should be part of our routine work (Nurse, Anaka hospital).

Most of the health workers strongly felt that IPV should be mainstreamed in health service service delivery with dedicated resources and spaces in the hospital. However, a few of the health workers thought that referral services could be offered from the hospitals where IPV victims are sign-posted to specialist areas. This study found that some of the health facilities had interventions in place to respond the IPV problem, for example Anaka Hospital has a unit for sexual gender based violence (SGV). However these were reported to be limited in scope and frequency as shown in the excerpt below:

IPV is only screened when a patient has got physical signs of violation and those with police cases (Doctor, Iganga hospital).

It is done occasionally, through history taking because sometimes they [victims] come with police forms (Doctor, Anaka hospital).

As nurses, screening is done but not as part of our duties and this is not frequent, it is only done when there 
are signs of violence but these cases are not followed up because we have no upper hand in settling the matters (Nurse, Iganga hospital).

As with most legal systems, in Uganda a physician's report is a mandatory requirement for some court cases such as those involving sexual abuse. Therefore health workers find themselves compelled to intervene at that level although it may not be part of their mainstream duties.

As a result of their continued interface with IPV victims, most of the health workers believed that they were competent and had the skills to screen for it. Some of the health workers had acquired relevant skills as part of their professional development:

We screen but we lack training in IPV, however some clinical officers have been trained in handling SGV victims (Doctor, Lacor hospital).

We have the capacity ...the hospital has a special unit in place though they need more human resources (Doctor, Anaka hospital).

Despite their positive attitudes towards screening for IPV the study participants were cognizant of their own limitations, particularly those related to the system and also other stakeholders involved such as victims, the communities within which they are embedded and other specialist workers. There were varied opinions regarding disclosure level and the general perception was that disclosure levels among IPV victims were found to be generally low. However, health workers also reported that victims, especially women, were increasing trusting health workers and disclosing, depending on the magnitude of the injury, in order to obtain help. This is in spite of the deep rooted fear and possible repercussion of their disclosure.

IPV victims never disclose and until the health worker sees a physical sign of violence then one can be asked and some of them still can't tell you what happened as they fear to be punished back home (Doctor, Iganga hospital).

They disclose to health worker. They are usually open to health workers more than anybody (Doctor, Gulu hospital).

The involvement of health workers is no solution because the communities are not aware of these IPV dangers so they may not stop them unless sensitized (Nurse, Iganga hospital).

The problem with Ministry of Gender is they develop policies but they don't implement or they don't sensitize the people then they just bring the policies to impose them on the people (Doctor, Anaka hospital).

\subsection{Views of Healthcare System Capacity to Screen/Involvement in IPV Screening}

At the individual level health workers were upbeat about screening for IPV. However they are also aware of the key role played by other stakeholders at the individual, household, community and national level which could have a significant impact on the efficacy of their intervention, as indicated in the above excerpts. A disconnection across these different levels and actors was cited as a major constraint to IPV screening.

The general consensus among this study's participants was that, despite health workers the health system had very limited capacity to effectively support IPV screening. The reason for this is not only lack of infrastructure but also resources in terms of time, human resources, and finances.

We are competent but not supported by the health system. We have the capacity however we lack time (Doctor, Gulu hospital).

The services are inadequate (Nurse, Anaka hospital).

We lack equipment to do IPV screening (Doctor, Iganga hospital).

\subsection{Conceptual Framework of Factors That May Hinder/Promote IPV Screening in Healthcare Uganda}

Discussants mentioned a number of factors that may deter/promote IPV screening. Congruent with the template used for this analysis, these factors appeared to follow a socio-ecological framework (Figure 1), where individual, care system and community/societal level factors were paramount.

\subsubsection{Individual-Level Factors}

Poor disclosure: Discussants' responses suggested that poor disclosure of abuse was to expect from clients. However there were implications in their statements that further probing by care providers was likely to lead to disclosure as pointed out in the excerpts below:

$I P V$ victims never disclose and until the health-worker sees a sign of physical abuse, then one can be asked and some of them still will not answer as they fear to be punished back at home (Doctor, Iganga hospital).

Victims disclose to health-workers mostly when they come for treatment of IPV injuries (Nurse, Lacor hospital).

They (women) all don't open up. However, sometimes the women blame the men yet they are the cause of the problem (Doctor, Gulu hospital).

Sometimes when they report, they are not sure you will give them a solution, so there is need to see how best to address the perpetrators of violence and what best ways to address the issue (Doctor, Anaka hospital).

Victims do not disclose, unless the health-worker probes further about the issue (Nurse, Gulu hospital).

As suggested in some of the excerpts above, it ap- 


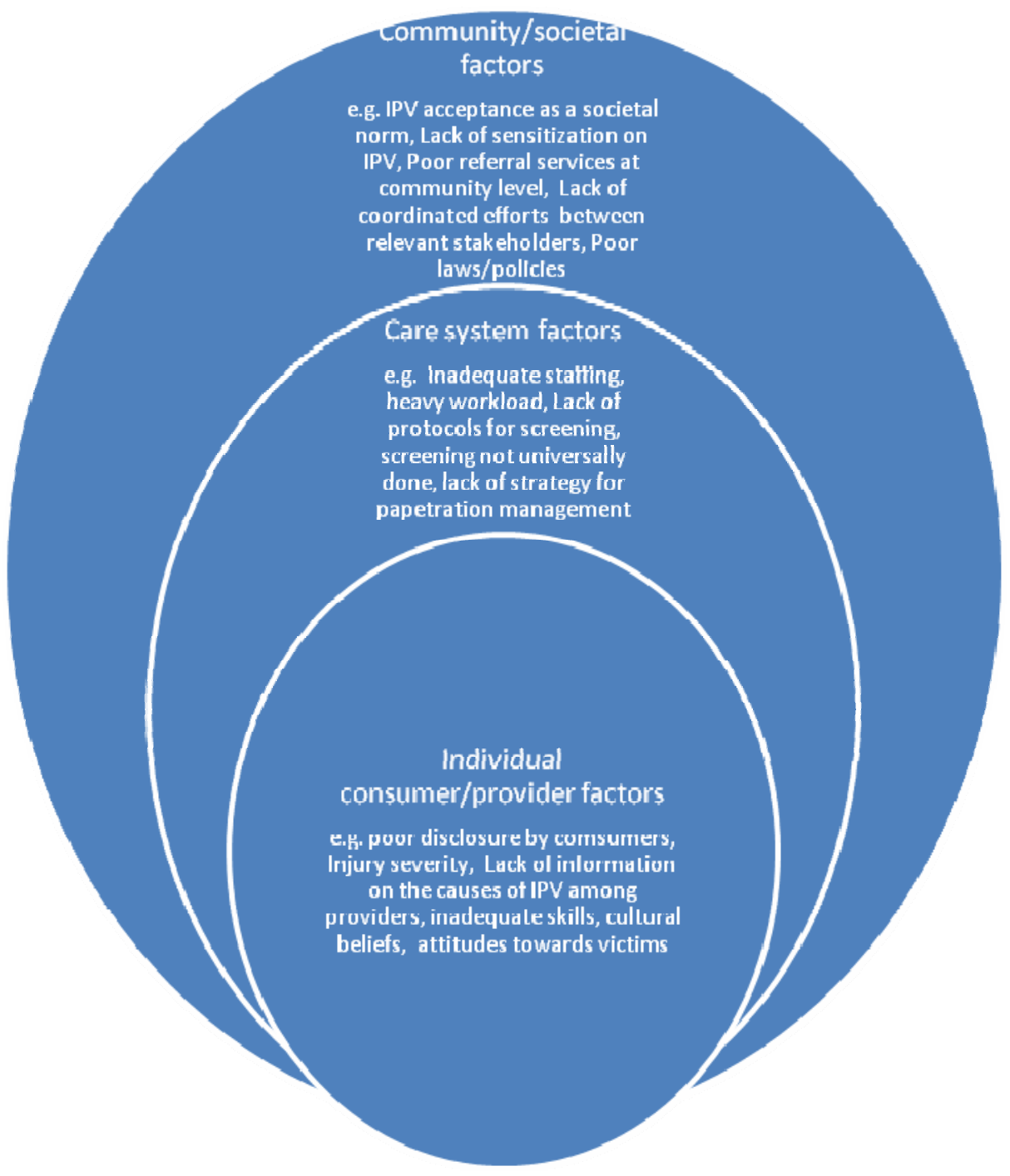

Figure 1. Model demonstrating factors that may hinder IPV screening in healthcare.

peared that IPV inquiry was often related to an observable injury. Moreover, in some responses there was a tendency by providers to blame the victim. Fear of retaliation from the abuser, levying the blame on the victim, and uncertainty regarding ability of healthcare workers to address the problem were some of the identifiable reasons for poor disclosure of/probing for IPV.

Poor skills and information on IPV: Poor skills in inquiry about IPV and a lack of information on IPV causes and management among care-providers were identified as potential barriers to IPV screening at the individual level as indicated in the responses below:

Health-workers need specific training in IPV screening (Doctor, Iganga hospital).

Health-workers are competent, but they have not been equipped to screen for IPV, so there is no capacity and they are not supported by the system (Nurse, Anaka hospital).
They (health-workers) need to be equipped with more knowledge and skills on IPV Screening (Nurse, Lacor hospital).

\subsubsection{Care System/Organisational Factors}

Understaffing, lack of time and protocols: Participants' responses indicated that under-staffing and lack of time could impact negatively on IPV screening, as suggested in the excerpts below. In addition, a lack of protocols for the purpose of screening and a weak support network were highlighted at the system level.

Health workers feel confident to screen, but we are understaffed (Doctor, Anaka hospital).

There is inadequate human resource (for screening) (Nurse, Anaka hospital).

They (health-workers) have the capacity to screen but they lack time (Nurse, Lacor hospital).

We lack equipment to do IPV screening (Doctor, 
Iganga hospital).

Creating links between health system and law keepers to find solutions to cases of IPV would be supportive in our role (Nurse, Iganga hospital).

\subsubsection{Community/Societal Level Factors}

At the community level, participants identified poor sensitization of community members, a culture of acceptance for IPV, a failure to coordinate efforts against IPV among relevant stakeholders and lack of policies on IPV management as crucial hinders to effective IPV screening, highlighted in the excerpts below:

All sectors in districts should pull efforts together and sensitize the community on all forms of IPV, its causes and consequences (Nurse, Iganga hospital).

The involvement of health workers is no solution because the communities are not aware of these IPV dangers so they may not stop them unless sensitized (Doctor, Iganga hospital).

Much as it is necessary to screen, so long as the cultural issues remain and women remain inferior to men, the screening will not be of great help (Nurse, Gulu hospital).

Most women in society don't even know their rights and they tend to be comfortable in their situation even when they are being tortured (Nurse, Gulu hospital).

Policies should be put in place so that the law can take its cause when people are battered (Nurse, Iganga hospital).

Creating links between health system and law keepers to find solution to cases (of IPV) would be supportive... (Nurse, Iganga hospital).

Not taking the identified IPV cases up by higher authorities like the police after screening and identification by health-workers is very frustrating when nothing is done to the perpetrators (Doctor, Lacor hospital).

\section{DISCUSSION}

The main objective of this qualitative study was to explore and deepen understanding on the perceptions and attitudes of healthcare professionals towards IPV screening, in order to develop a conceptual model for factors likely to hinder/promote IPV screening in healthcare Uganda. Congruent with what has been reported in quantitative studies $[31,32,35]$ we found that a complex interaction of factors at individual, organisational and societal levels may influence screening for IPV in healthcare Uganda, despite the fact that healthcare professionals were generally positive and supportive towards interventions to screen for IPV in healthcare.

First, at the individual level, healthcare workers exhibited a narrow conceptualisation of IPV and related it mainly to physically observable phenomena. It is highly likely that this constricted definition would negatively impact on their capacity to effectively detect and respond to IPV. As key frontline workers in service delivery, doctors and nurses therefore need to be supported to fully understand both subtle and explicit manifestations of IPV. In addition, poor knowledge of the epidemiology of IPV and skills in its detection were reported to potentially influence IPV screening. Related to poor skills, individual fears of addressing IPV with victims and perpetrators were signalled in the responses. Moreover, healthcare providers faced challenges of prompting unwilling patients to disclose exposure to IPV. These factors coupled by acceptance of IPV as a means to punish women who transgress from societal gender norms at the individual and societal levels, potentially render the practice of IPV screening difficult. Thus, it is important that before any screening program is introduced in healthcare Uganda, a comprehensive training package comprising understanding of the conceptualisation and epidemiology of IPV, the modalities involved in IPV screening per se, and tackling patriarchal/gender biased attitudes among individual care providers is warranted.

Secondly, at the organisational level, understaffing, inadequate human resource and capacity for IPV screening were discussed by participants as potential hinders of IPV screening, consistent with quantitative studies reported in other dissimilar societal context like Nigeria [32] and Sweden [35]. Recent statistics in Uganda [MoH 2011; 2009] have demonstrated an understaffing crisis in the country's health system of up to $50 \%$, resulting in a heavy workload and consequent poor service delivery at the frontline. This, coupled by the quest from discussants for incorporation of screening protocols and other modalities (e.g. strategy for perpetration management) demonstrate a low level of system preparedness to respond to IPV screening currently in Ugandan healthcare. Whereas clinical and related skills are invaluable, health workers need to be supported by an organisation that is fully fledged to meet the demands of patients, including IPV victims. Thus the implications of these results on the re-organisation and resourcing of the healthcare arena need not be over-emphasised if IPV screening is to develop into an effective practice in Ugandan healthcare.

Participants reported seemingly major barriers to IPV screening at the societal level. IPV was found to be deeply entrenched in cultural practices, decision making and resultant action in male hegemonic societies. In trying to explore the places and relationships in which women's lives were embedded, it was found that such societies invite oppressive monitoring and control on women and these serve to further entrench the vulnerability of women. As a result of this cultural hegemony, IPV becomes largely engendered at all levels of society from interpersonal relationships to macro level decisions 
on the same. It was perhaps not surprising that this study found casual "acceptance" of some form of IPV such as occasional wife beating - even by healthcare professionals purported to advocate for IPV screening. Together with previous findings from quantitative studies demonstrating significant societal acceptance of abuse of women for "failure" in normative domestic roles [26,27], these findings drum the need for a concerted awareness campaign in the general population of the health and societal defects resulting from IPV, as such education is known to modify distorted attitudes and reduce practices that are harmful to health such as IPV [44]. Also at the societal level, discussants expressed concerns over a lack of reliable referral services to which eventual IPV victims could be referred and drummed the need for coordinated efforts between different stakeholders in society and healthcare system if IPV screening in healthcare is to be effective.

\section{Implications for Practice, Limitations and Further Research}

In summary, this study provided valuable information prior to the initiation of IPV screening in Uganda. It is recommended that before a routine screening protocol is introduced the following conditions are paramount including: 1) comprehensive staff training addressing the conceptualisation of IPV, its epidemiology and modalities involved in screening is warranted. It is hoped that such training may modifying individual health-workers' attitudes towards IPV; 2) organisational changes responding to understaffing issues and availing clear and comprehensive screening protocols for IPV are necessary; 3) awareness campaigns of the negative impact of IPV on society e.g. health effects (running parallel with the above recommendations) are crucial to modify attitudes towards IPV in the society, as well as prepare the general population to will-fully disclose IPV to health-workers; 4) building a rigorous network between the healthcare system itself and other community advocates in IPV prevention may improve among others referral services for IPV victims.

The limitations of the current study deserve some acknowledgement. First, applicability of the findings to Ugandan healthcare in general should be done with caution. The study was carried out in only 3 of the countries' 111 districts and in 2 of the 4 main regions of Uganda (i.e. northern and eastern Uganda). However, in Uganda, the structure, policy and activities of hospitals appear to vary depending on whether the hospital is a regional, district or other smaller care units. In that respect therefore, the findings could be seen to represent views on IPV screening in referral and district hospitals in northern and eastern Uganda. This notwithstanding, our findings generated a conceptual framework for factors possi- bly influencing IPV screening, hypotheses of which could be tested using quantitative methods in future related research in Uganda. A Second limitation concerns a possible lack of heterogeneity in the FGDs. We did not perform mixed FGDs in heterogeneous groups of professionals (i.e. including both nurses and doctors). Though this was initially planned for, it came to our attention that there remains a hierarchy in position between nurses and doctors in Uganda. Thus, the nursing staff argued that they were unable to discuss these issues freely in the presence of doctors (assumed to be higher in hierarchy). The consequences of the lack of heterogeneity in FGDs with regard to staff cadre on the results thus are difficult to predict. It is plausible that mixed groups could have enriched the discussions, providing impetus for the emergence of new themes otherwise undetectable in homogenous groups alone. Finally, the views of nursing assistants, aides, midwives and other staff were not sought in this study. Yet they are of importance in the healthcare of potential victims of IPV. This omission was due to the lack of such cadres in some of the smaller hospitals studied. Future research should find modalities for incorporation of these groups, to achieve a holistic view of healthcare providers' perceptions on screening for IPV in healthcare, Uganda. Moreover, the views of other stakeholders particularly the potential victims and perpetrators on IPV screening in healthcare call for a separate study on its own right.

\section{ACKNOWLEDGEMENTS}

We are most grateful to the Swedish Council for Working Life and Social Research (FAS) and the Marie-curie program for funding this study as part of the COFAS program.

\section{REFERENCES}

[1] World Health Organization (2002) World report on violence and health. WHO, Geneva.

[2] Mwenesi, B.K., Buluma, R.C.B., Kong'ani, R.U. and Nyarunda, V.M. (2003) Gender violence. Kenya Demographic and Health Survey, Final Report.

[3] Gage, A. (2005) Women's experience of Intimate Partner Violence in Haiti. Social Science \& Medicine, 61, 343364. doi:10.1016/j.socscimed.2004.11.078

[4] Koenig, M.A., Lutalo, T., Zhao, F., Nalugoda, F., Wabwire-Mangen, F., Kiwanuka N., et al. (2003) Domestic violence in rural Uganda: Evidence from a community-based study. Bulletin of the World Health Organization, 81, 53-60.

[5] Campbell, J. (2002) Health consequences of Intimate Partner Violence. Lancet, 359, 1331-1336. doi:10.1016/S0140-6736(02)08336-8

[6] Emenike, E., Lawoko, S. and Dalal, K. (2008) Intimate Partner Violence and Reproductive health of women in Kenya. International Nursing Review, 55, 97-102. 
doi:10.1111/j.1466-7657.2007.00580.x

[7] Koenig, M.A., Lutalo, T., Zhao, F., Nalugoda, F., Wabwire-Mangen, F., Kiwanuka N., et al. (2003) Domestic violence in rural Uganda: Evidence from a community-based study. Bulletin of the World Health Organization, 81, 53-60.

[8] Garcia-Morena, C., Jansen, H., Ellsberg, M., Heise L. and Watts, C. (2005) WHO Multi-country study on women's health and domestic violence against women: Initial results prevalence, health outcomes and women's responses. WHO, Geneva.

[9] Emenike, E, Lawoko, S. and Dalal, K. (2008) Intimate Partner Violence and Reproductive health of women in Kenya. International Nursing Review, 55, 97-102. doi:10.1111/j.1466-7657.2007.00580.x

[10] Plichta, S.B. (2004) Intimate Partner Violence and physical health consequences: Policy and practice implications. Journal of Interpersonal Violence, 19, 1296-323. doi:10.1177/0886260504269685

[11] (1992) American Medical Association diagnostic and treatment guidelines on domestic violence. Archives of Family Medicine, 1, 39-47.

[12] Furniss, K., McCaffrey, M., Parnell, V. and Rovi, S. (2007) Nurses and barriers to screening for Intimate Partner Violence. MCN: The American Journal of Maternal/Child Nursing, 32, 238-243. doi:10.1097/01.NMC.0000281964.45905.89

[13] John, I.A., Lawoko, S. and Oluwatosin, A. (2011) Acceptance of screening for Intimate Partner Violence, actual screening and satisfaction with care amongst female clients visiting a health facility in Kano, Nigeria. African Journal of Primary Health Care \& Family Medicine, 3, 6 Pages. doi:10.4102/phcfm.v3i1.174

[14] Erikson, M.J., Hill, T.D. and Siegal, R.M. (2001) Barriers to domestic Violence screening in the Padiatric setting. Pediatrics, 108, 98-102. doi:10.1542/peds.108.1.98

[15] Roelens, K., Verstraelen, H., Van Egmond, K. and Temmerman, M. (2006) A knowledge, attitudes and practice survey among obstetrician-gynaecologists on Intimate Partner Violence in Flanders, Belgium. BMC Public Health, 6, 238. doi:10.1186/1471-2458-6-238

[16] Abbott, J., Johnson, R., Koziol-McLain, J., et al. (1995) Domestic violence against women: Incidence and prevalence in an emergency department population. Journal of the American Medical Association, 273, 1763-1767. doi:10.1001/jama.1995.03520460045033

[17] Thomas, P. and Lowitt, N.R. (1995) Clinical problem solving: A traumatic experience. The New England Journal of Medicine, 333, 307-310. doi:10.1056/NEJM199508033330509

[18] Synder, J.A. (1994) Emergency department protocols for domestic violence. Journal of Emergency Nursing, 20, 65-68.

[19] Krug, E.G., et al. (2002) World report on violence and health. World Health Organization, Geneva.

[20] Campbell, J. (2002) Health consequences of Intimate Partner Violence. Lancet, 359, 1331-1336.

\section{doi:10.1016/S0140-6736(02)08336-8}

[21] Okenwa, L., Lawoko, S. and Jansson, B. (2009) Factors associated with disclosure of Intimate Partner Violence among Women in Lagos Nigeria. International Journal of Injury and Violence Research, 1, 37-47. doi:10.5249/jivr.v1i1.15

[22] Petersen, R., Gazmararian, J. and Clark, K. (2001) Partner violence. Implications for health and community settings. Women's Health Issues, 11, 116-125. doi:10.1016/S1049-3867(00)00093-1

[23] World Health Organisation (2005) WHO multi-country study on women's Health and domestic violence against women. WHO, Geneva.

[24] Brottsförebygande Rådet (BRÅ) (2009) Våld mot kvinnor och män i nära relationer: Våldets karaktär och offrets erfarenhet av kontakter och rättsväsendet (In Swedish). BRÅ Rapport, 12.

[25] Ramsey, J., Richardson, J., Carter, Y.H., Davidson, L. and Feder, G. (2002) Should health professionals screen women for domestic violence? Systematic review. British Medical Journal, 2, 314.

[26] Lawoko S. (2006) Factors associated with attitudes towards violence: A study of women in Zambia. Victoria Silvstedt, 21, 645-656.

[27] Uthman, O.A., Lawoko, S. and Moradi, T. (2009) Factors associated with attitudes towards Intimate Partner Violence against women: A comparative analysis of 17 subSaharan countries. BMC International Health and Human Rights, 9, 14. doi:10.1186/1472-698X-9-14

[28] Erikson, M.J., Hill, T.D. and Siegal, R.M. (2001) Barriers to domestic violence screening in the Padiatric setting. Pediatrics, 108, 98-102. doi:10.1542/peds.108.1.98

[29] Roelens, K., Verstraelen, H., Van Egmond, K. and Temmerman, M. (2006) A knowledge, attitudes and practice survey among obstetrician-gynaecologists on intimate partner violence in Flanders, Belgium. BMC Public Health, 6, 238. doi:10.1186/1471-2458-6-238

[30] Waalen, J., Goodwin, M., Alison, M., Spitz, M.S., Petersen, R. and Saltzman, L.E. (2000) Screening for Intimate Partner Violence by health care providers: Barriers and interventions. American Journal of Preventive Medicine, 19, 230-237. doi:10.1016/S0749-3797(00)00229-4

[31] Maiuro, R.D., Vitaliano, P.P., Sugg, N.K., Thompson, D.C., Rivara, F.P. and Thompson, R.S. (2000) Development of a health care provider survey for domestic violence: Psychometric properties. American Journal of Preventive Medicine, 19, 245-252. doi:10.1016/S0749-3797(00)00230-0

[32] John, I., Lawoko, S. and Svanstrom, L. (2011) Screening for Intimate Partner Violence in healthcare in Kano, Nigeria: Extent and determinants. Journal of Family Violence, 26, 109-116. doi:10.1007/s10896-010-9348-y

[33] South Eastern Sydney Area Health Service (SESAHS) (1997) Domestic violence policy and protocol. SESAHS, Caringbah.

http://www.ciap.health.nsw.gov.au/hospolic/domviol/dom esticviolence. html

[34] Gerbert, B., Abercrombie, P., Caspers, N., Lowe, C. and 
Bronstone, A. (1999) How health care providers help battered women: The survivor's perspective. Women \& Health, 29, 115-135. doi:10.1300/J013v29n03_08

[35] Lawoko, S., Sanz, S, Helstrom, L. and Castren, M. (2011) Screening for Intimate Partner Violence against women in healthcare Sweden: Prevalence and determinants. ISRN Nursing, 2011, Article ID 510692, 7 Pages. doi: $10.5402 / 2011 / 510692$

[36] Green, J. and Thorogood, N. (2004) Qualitative methods for health research. SAGE, London.

[37] Denzin, N.K. and Lincoln, Y.S, (1994) Handbook of qualitative research. SAGE, London, ix.

[38] King, N. (2004) Using templates in the thematic analysis of text. In: Cassels, C. and Symon, G, Eds., Essential Guide to Qualitative Methods in Organizational Research, Sage, London, 256-270.

[39] Cassels, C. and Symon, G. (2004), Essential guide to qualitative methods in organizational research. Sage, London, 256-270.
[40] Ochen, E.A. (2011) Life beyond the bush: Examining the challenges and opportunities for reintegration of formerly abducted child mothers in northern Uganda. PhD Thesis, University of Huddersfield, Queensgate.

[41] Troung, Y. and Simmons, G. (2010) Perceived intrusiveness in digital advertising: Strategic marketing implications. Journal of Strategic Marketing, 18, 239-256. doi:10.1080/09652540903511308

[42] Dries, N. and Pepermans, R. (2008) “Real” high potential careers. Personnel Review, 37, 85-108. doi:10.1108/00483480810839987

[43] Wainright, D.W. and Waring, T.S. (2007) The application and adaptation of a diffusion of innovation framework for information systems research in NHS general medical practice. Journal of Information Technology, 22, 44-58. doi:10.1057/palgrave.jit.2000093

[44] Wolf, D.A. and Jaffe, P.G. (1999) Emerging strategies in the prevention of domestic violence. Domestic Violence and Children, 9, 133-144. 